\title{
Opinião de idosos trabalhadores sobre sua qualidade de vida
}

\section{Elderly workers' opinion about their quality of life}

DOI: $10.46919 / \operatorname{archv2n3-016}$

Recebimento dos originais: 01/01/2021

Aceitação para publicação: 31/03/2021

\section{Felipe Queiroz Dias Rocha}

Mestre pela Faculdade de Psicologia e de Ciências da Educação da Universidade do Porto (FPCEUP) Instituição Atual: sem trabalho, mas a FPCEUP foi a última instituição de ensino em que estudei (caso possa substituir o trabalho).

Endereço: Rua Tabajaras, 100 apto 51A - 03121-010 - Mooca, São Paulo/SP

E-MAIL: felipequeiroz.dr@gmail.com

\section{Marcelo Arruda Piccione}

Especialista pelo Instituto Sedes Sapientiae

Instituição Atual: Sport Club Corinthians Paulista

Endereço: Rua Arlindo Béttio, 502 - Trabalhadores, São Paulo - SP, 03828-000, Brasil

E-MAIL: piccione_marcelo@hotmail.com

\section{RESUMO}

No mundo inteiro o número de idosos tem crescido. A velhice traz consequências psíquicas, físicas e sociais. Assim, muitas ponderações são levantadas sobre como a sociedade reagirá ao envelhecimento. Objetivou-se observar a qualidade de vida dos idosos trabalhadores e qual a influência dessas atividades em outras áreas de suas vidas. Foram coletados dados de 36 idosos de ambos os sexos que trabalham atualmente; os dados foram obtidos a esmo, para isso, foram utilizados 36 questionários com 46 questões fechadas e duas abertas. Aplicou-se o teste do Qui-quadrado para descobrir se existe diferença estatisticamente significante entre as variáveis. Destaca-se que 77,77\% Discordam Totalmente da premissa de que não apreciam muito a vida; 66,66\% Discordam Totalmente da hipótese de não receberem força pessoal e apoio provindo de Deus; $44,44 \%$ dizem aproveitar a vida Mais ou menos, $\chi_{\mathrm{o}}^{2}=3,99$ e $\chi_{c}^{2}=9,48$, não havendo diferença estatisticamente significante; 41,66\% estão Satisfeitos com seu acesso aos serviços de saúde, $\chi \mathrm{o}^{2}=4,20$ e $\chi^{2} c=9,48$, não existindo diferença estatisticamente significante; $36,11 \%$ dizem-se Satisfeitos com seu meio de transporte, $\chi \mathrm{o}^{2}=2,20$ e $\chi_{c}^{2}=7,81$, não havendo diferença estatisticamente significante. Conclui-se que os idosos trabalhadores estão satisfeitos com a maioria dos aspectos biopsicossociais que envolvem sua vida.

Palavras-chave: velhice, envelhecimento, biopsicossociais.

\begin{abstract}
Worldwide the number of elderly people is growing. Old age brings psychic, physical, and social consequences. Thus, many ponderings are raised about how society will react to aging. We aimed to observe the quality of life of elderly workers and what influence these activities have on other areas of their lives. Data were collected from 36 elderly people of both genders who are currently working; the data were obtained randomly, and for this, 36 questionnaires with 46 closed and two open questions were used. The
\end{abstract}


Chi-square test was applied to find out if there is a statistically significant difference between the variables. It is noteworthy that $77.77 \%$ Totally Disagree with the premise that they do not enjoy life very much; 66.66\% Totally Disagree with the hypothesis that they do not receive personal strength and support from God; $44.44 \%$ say they enjoy life More or less, $\chi_{o}^{2}=3.99$ and $\chi^{2}=9.48$, with no statistically significant difference; $41.66 \%$ are satisfied with their access to health services, $\chi \mathrm{o}^{2}=4.20$ and $\chi^{2} c=9.48$, with no statistically significant difference; $36.11 \%$ say they are satisfied with their means of transportation, $\chi \mathrm{o}^{2}=2.20$ and $\chi \mathrm{o}^{2}=7.81$, with no statistically significant difference. It is concluded that the working elderly are satisfied with most of the biopsychosocial aspects surrounding their life.

Keywords: old age, aging, biopsychosocial.

\section{INTRODUÇÃO}

No mundo inteiro, à medida que os anos vão passando, o número de idosos tem crescido. $\mathrm{O}$ fenômeno está relacionado a vários fatores, como a queda da taxa de natalidade e o aumento da expectativa de vida; ambos, por sua vez, decorrentes de causas distintas. No Brasil a situação não é diferente e pouco a pouco o mito de que somos um país de jovens vai sendo derrubado. Em 2025, segundo a Organização Mundial da Saúde (OMS), em 26 países a esperança de vida deverá ser de 80 anos. Estão no topo da lista dos países com população de maior longevidade a Islândia, a Itália, o Japão e a Suécia (Neri, 2001).

A percepção de um indivíduo quanto a sua posição na vida no que tange a seus valores, concepções e conceitos da cultura da sociedade onde vive concernente às suas metas, objetivos e preocupações é o que definimos como qualidade de vida. Ela envolve três vertentes diferentes, mas essenciais, de acordo com a OMS: o fato de ser um conceito subjetivo, multidimensional e inclusivo de pontos característicos negativos e positivos (Trentini, Xavier \& Fleck, 2006).

O conceito de qualidade de vida possui inúmeros significados nas diversas áreas existentes. Por exemplo, no campo da Economia, qualidade de vida está relacionada com medidas, como a renda per capita, pois indica o acesso das camadas sociais aos serviços de educação, saúde, habitação, entre outros mais. No campo da Política e da Sociologia, o foco é dado diretamente a uma população, não de forma individual. Na Psicologia Social, o nível de satisfação é o conceito mediatizador da experiência subjetiva, que é a maior referência, por sua vez (Trentini, Xavier \& Fleck, 2006).

É por isso também que houve uma valorização muito grande da qualidade de vida na fase do envelhecimento nos últimos 30 anos. Existem três tipos distintos de se envelhecer: com patologia, de forma normal ou de modo bem-sucedido (Trentini, Xavier \& Fleck, 2006).

A velhice com patologia é denotada quando há uma síndrome, doença ou deficiência crônica típica do envelhecimento, limitando severamente as atividades normais do indivíduo idoso. Nesse ponto, as 
funções que o sujeito a esse tipo de velhice desempenhava enquanto adulto jovem são perdidas ou se tornam menos nítidas (Trentini, Xavier \& Fleck, 2006).

Velhice normal ou usual é o oposto da velhice com patologia, ou seja, é o contrário do processo de envelhecimento acompanhado de doenças que trazem limitações graves. Na velhice usual é estritamente comum o surgimento de doenças mentais e/ou físicas, mas com um teor leve ou moderado em sua manifestação, provocando apenas mudanças parciais no quotidiano dos idosos (Trentini, Xavier \& Fleck, 2006).

Velhice bem-sucedida ou ótima é a velhice com a preservação do estado físico de saúde como dos adultos jovens, tomando um estado bom ou ótimo com respeito ao bem-estar social e pessoal como exemplo (Trentini, Xavier \& Fleck, 2006).

Envelhecer pressupõe sofrer alterações físicas, psicológicas e sociais. Tais alterações são naturais e gradativas. É importante salientar que essas transformações são gerais, podendo se verificar em idade mais precoce ou mais avançada e em maior ou menor grau, de acordo com as características genéticas de cada indivíduo e, principalmente, com o modo de vida de cada um. A alimentação adequada, a prática de exercícios físicos, a exposição moderada ao Sol e a estimulação mental são alguns fatores que podem retardar ou minimizar os efeitos da passagem do tempo. Com o passar dos anos, o desgaste é inevitável. Sabemos que a velhice não é uma patologia, mas, sim, uma fase na qual o ser humano fica mais suscetível a doenças. É uma época na qual as pessoas adoecem mais (Zimerman, 2000).

O envelhecimento biológico, então, é um fenômeno singular em cada idoso, e não uma doença; até porque as patologias que se dão nesse período do desenvolvimento humano são diagnosticáveis e tratáveis. E ainda é possível que os idosos vivam bem e com qualidade mesmo que seu funcionamento orgânico esteja fragilizado ou deficitário (Martins, Albuquerque, Nascimento, Barra, Souza \& Pacheco, 2007).

Mas o estado de saúde não deixa de ser um dos fatores que pode influenciar na aposentadoria antecipada. Uma saúde deficitária aumenta a probabilidade de aposentadoria. Provavelmente, os que se aposentam mais tarde gozam de boa saúde (Bee, 1997).

Segundo Colalto (2002), somente $40 \%$ dos idosos estão satisfeitos com o serviço de saúde que possuem.

Nesse ponto, eles deixaram bem claro de forma unânime em expressar que estão insatisfeitos com o atendimento porque os médicos não lhes dão tempo para falar de seus problemas, não fazem perguntas e às vezes nem levantam seu olhar para eles (Beres, 1994).

O fato é que os idosos precisam utilizar estratégias que os possibilitem ter uma velhice satisfatória. Isto implica em mudar seus hábitos, engajar-se em atividades produtivas, realizar seus projetos de vida, ingressar em universidade de terceira idade, desenvolver serviços voluntários, dentre tantas outras iniciativas possíveis. Enfim, um envelhecimento bem-sucedido depende de como o idoso vai enfrentar os 
desafios da vida, lutar pelos seus direitos de cidadão e colocar em prática projetos viáveis dentro das condições pessoais e do meio ambiente em que vive (Martins et al., 2007).

A satisfação com a vida é uma das medidas do bem-estar psicológico que reflete a avaliação pessoal do indivíduo sobre determinados domínios. Dois aspectos essenciais do bem-estar psicológico são a capacidade de acomodação às perdas e de assimilação de informações positivas sobre o self, um sistema composto por estruturas de conhecimento sobre si mesmo e um conjunto de funções cognitivas que integram ativamente essas estruturas ao longo do tempo e de várias áreas do funcionamento pessoal (Neri, 2001).

Entrementes, Zimerman (2000) diz que 15\% dos idosos necessitam de atendimento em saúde mental e $2 \%$ das pessoas com mais de 65 anos apresentam quadros de depressão que, muitas vezes, não são percebidos pelos familiares e cuidadores, sendo encarados como características naturais do envelhecimento.

$\mathrm{Na}$ fase pós-menopausa, ocorre nas mulheres alterações nos genitais, na aparência corporal e modificações durante as relações sexuais. Estas mudanças são observadas pelos seguintes sintomas: a pele fica mais seca, a lubrificação vaginal diminui e a mucosa vaginal atrofia. Por causa da baixa lubrificação, a mulher pode sentir dor na hora da penetração. Porém, esses sintomas não reduzem a libido nem a capacidade orgástica, mas alguns fatores podem interferir no ciclo de resposta sexual, entre eles o psicológico e o social. Tudo isso pode acarretar mudanças na imagem que a mulher tem de seu próprio corpo e das modificações que ocorrem, achando-se menos atraente e incapaz de conquistar seu parceiro, gerando assim, conflitos emocionais (Veras, 1995).

Os homens também sofrem com o envelhecimento, por volta dos 50-70 anos, na fase de excitação, a ereção não se estabelece tão rapidamente como na juventude e muitas vezes há necessidade de manipulação física de seu órgão genital pela parceira. Se essa aquisição da ereção for observada de forma negativa e não como um processo natural do envelhecimento, o homem poderá sentir-se incapaz de continuar sua atividade sexual, gerando assim uma possível impotência. A falta de informação sobre essas alterações no exercício da sexualidade pode fazer com que o homem tenha uma visão negativa e deixe de exercer a atividade sexual (Veras, 1995).

Rabelo, Souza, Gomes, Fujimoto, Mota e Oliveira (2008) dizem que os jovens (principalmente as mulheres) têm perspectivas positivas sobre a capacidade de cognição, as relações pessoais, a autonomia e a autoimagem dos idosos e consideram possível ser feliz na velhice. Por outro lado, demonstram-se bastante temerosos quanto ao seu próprio envelhecimento, chegando a declarar que é preferível morrer que sofrer a angústia e a solidão da velhice, pois acreditam que a terceira idade, em contrapartida, prenuncia a chegada de dependência, solidão e morte.

Com isso, muitas ponderações têm sido levantadas sobre como o envelhecimento humano será visto e considerado pelas sociedades (Veloz, Nascimento-Schulze \& Camargo, 1999). 
De acordo com Araújo (1999), o aspecto religioso também tem grande influência nessa fase da vida, de forma que $100 \%$ da população pesquisada demonstrou afinidade com algum tipo de atividade sacra. Quanto às razões para a ocorrência desse fato, verifica-se que a prática de uma religião pelo idoso permitelhe estabelecer um elo entre as limitações e suas potencialidades ou, quando isso não ocorre, ajuda-o a vencer com mais facilidade esse estágio final da vida.

Os ritos (atos) e os mitos (falas) estão na base da construção de todas as religiões, e são eles, portanto, os demonstradores da criação de símbolos para a expressão do divino em nós. Assim, a religião pode ser definida como o reconhecimento das realidades mais elevadas que a consciência não consegue compreender e, quando levada à plena fruição psicológica, produz a unidade interior e a totalidade do ser humano (Baptista, 2003).

Não há diferença entre os estilos de vida levados pelos idosos no que tange a prática de orações, a maior parte deles reza por propósitos como: saúde física, obtenção de paz ou amor, por problemas familiares, por gratidão, entre outros (Orlando, Dias, Brasil, Araújo \& Buriti, 2008).

Segundo Diniz (2003), a religião é uma experiência que nos toca e nos modifica. Ela tem que ser transformadora. Não se trata de religião como algo concreto, que possui credo, moral e normas, mas sim de uma fé, que significa o encontro vivo com Deus.

Panzini, Rocha, Bandeira e Fleck (2007) dizem que a felicidade está associada à frequência em serviços religiosos e que a crenças influenciam de forma significativa na visão que o sujeito constrói do mundo. A religião pode explicar o propósito de vida e promover bem-estar.

Aliás, à medida que os indivíduos amadurecem o senso de significado positivo, de existência de um verdadeiro propósito na vida (o qual é subjetivo) e de tranquilidade concernente ao futuro aumenta e supera o dos jovens (Freitas, 2020).

O sentido e a apreciação da vida influenciam o bem-estar e elevam em 33\% os sentimentos e atitudes relativas à: aceitação de si, relações interpessoais sadias, crescimento pessoal, domínio do ambiente e propósito, embora acresçam muito pouco à satisfação com a vida e ao surgimento de emoções positivas, assim como também diminuem pouquíssimo as emoções negativas. Já a tranquilidade quanto ao futuro também influencia o bem-estar e ainda maximiza substancialmente os fatores emocionais positivos e de satisfação com a vida, enquanto as emoções negativas diminuem quando há essa despreocupação e sentimentos. Por outro lado, os elementos relativos à percepção que os indivíduos têm do seu próprio envolvimento com as adversidades da vida e de seu grau de funcionamento psicológico observados em: atitudes de aceitação de si, relações interpessoais sadias, crescimento pessoal, controle do meio e propósito, se elevam pouquíssimo (Freitas, 2020).

Os sujeitos que encontram sentido em suas vidas têm mais habilidades sociais e estão mais abertos para distinguir e buscar o apoio social adequado quando necessário - a felicidade ou bem-estar advém do 
sentido existencial atribuído à vida. Esse sentido é responsável por influir de forma sensível e positiva no desenvolvimento psicológico humano e no modo como os indivíduos defrontam suas adversidades. É importante às pessoas acreditarem em um futuro promissor a fim de obterem forças diante dos desafios inerentes da vida e de diminuir os níveis de ansiedade e de outros sintomas relativos ao porvir (Freitas, 2020).

Assim é percebida a importância de indivíduos maduros, como idosos, nas empresas, os quais contribuem com o desenvolvimento, com o equilíbrio emocional e com o encontro do sentido que favoreça o enfrentamento mais assertivo de demandas da organização (Freitas, 2020).

\section{OBJETIVOS}

\subsection{OBJETIVO GERAL}

Esta pesquisa tem por objetivo verificar como é a qualidade de vida dos idosos que exercem atividade de trabalho mesmo após o período da aposentadoria e qual a importância que essas atividades têm em suas vidas.

\subsection{OBJETIVOS ESPECÍFICOS}

Os objetivos específicos são:

- Observar como os idosos se sentem trabalhando após terem atingido a idade oficial que os permite aposentar;

- Verificar se há influência dessas atividades de trabalho nas diversas áreas de suas vidas.

\section{MÉTODO}

\subsection{PARTICIPANTES}

A coleta de dados foi feita com 36 idosos de ambos os sexos - sendo que 88,90\% eram homens e $11,10 \%$ eram mulheres - que tinham idade igual ou superior a 65 anos e que estivessem trabalhando atualmente de forma registrada ou autônoma e de modo constante ou inconstante. A idade média é 71,5 anos, a mediana é 71 anos, o desvio padrão é de 5,4 e a amplitude de idade é de 22 anos (a menor idade é 65 e a maior é 87).

\subsection{MATERIAL}

Foram utilizados questionários WHOQOL; a escolha desse material se deu por ser ele um instrumento reconhecido internacionalmente para se avaliar qualidade de vida (conforme a opinião dos participantes). Ele investiga seis domínios diferentes da vida dos sujeitos: físico, psicológico, meio ambiente, nível de independência, relações pessoais e crenças. 
O questionário continha três partes distintas. A primeira era a caracterização do indivíduo, onde eram feitas perguntas sobre si e sua vida particular. Na segunda parte havia questões fechadas sobre diversos aspectos que envolvem a qualidade de vida dos idosos. Cada questão tinha cinco variáveis, sendo que sob elas havia uma sequência numérica ordenada de um a cinco. A última parte continha frases afirmativas e negativas que diziam respeito à opinião do sujeito participante quanto à veracidade daquela frase em seu ponto vista ou não, ou seja, havia frases em que o sujeito relatava com que intensidade concordava ou não com elas. As frases eram concernentes às expectativas futuras do indivíduo e a influência que a crença em Deus tem sobre sua vida.

\subsection{PROCEDIMENTOS}

Primeiramente nos apresentávamos como pesquisadores e explicávamos o intuito da pesquisa. Em seguida, se o sujeito estivesse dentro dos critérios estabelecidos e concordasse em participar, lhe introduzíamos o Termo de Consentimento Livre e Esclarecido (TCLE) e o questionário, lhe tirando qualquer dúvida eventual ou lhe esclarecendo as entrelinhas do termo.

Os dados foram coletados acidentalmente, ou seja, os idosos que estavam nas condições referidas (trabalhando e que tinham 65 anos de idade ou mais) foram entrevistados pessoalmente, sendo abordados pelos pesquisadores em qualquer circunstância viável, isto é, num horário pertinente em que podiam participar da pesquisa, sem lhes causar prejuízo. Alguns, por exemplo, foram abordados durante um momento de ócio, outros durante o trabalho e outros foram agendados com antecedência.

Dos voluntários, 15 foram entrevistados na Mooca, seis no Brás, outros seis na Sé, cinco na Zona Cerealista, três na Vila Mariana e um no Cambuci.

O projeto foi registrado no parecer 017/2005 do Comitê de Ética de Pesquisas (CEP) com seres humanos, em Brasília, e na CAAE 005.0.237.000.05.

\section{RESULTADOS E DISCUSSÃO}

Os resultados foram organizados em tabelas de distribuição e frequência e analisados de forma qualitativa e quantitativa. Para a comparação dos dados foi utilizado o teste não paramétrico do Quiquadrado.

Tabela 1 - Autoavaliação da qualidade de vida

\begin{tabular}{lcc}
\hline Autoanálise & F & \% \\
\hline Ruim & 2 & 5,55 \\
Nem ruim nem boa & 14 & 38,88 \\
Boa & 17 & 47,22 \\
Muito boa & 3 & 8,33 \\
\hline Total & $\mathbf{3 6}$ & $\mathbf{1 0 0}$ \\
\hline
\end{tabular}


Na Tabela 1, percebe-se que a qualidade de vida levada pelos idosos é classificada como Boa pela maior parte deles $(47,22 \%)$ e é considerada como Nem ruim nem boa por 38,88\%. Nota-se, ainda, que $8,33 \%$ consideram sua qualidade de vida Muito boa e apenas 5,55\% a classificam como Ruim. Nenhum idoso optou pela alternativa Muito ruim, o que fez com que esta variável fosse eliminada da tabela.

Para verificar se existe diferença estatisticamente significante foi aplicado o teste do Qui-quadrado, sendo $\chi \mathrm{o}^{2}=0,28$ e $\chi^{2}=7,81$, não havendo, assim, diferença estatisticamente significante; também se considera n.g.l. $=3$ e e $\alpha=0,05$.

Na Tabela 2, se observa que 44,44\% dos participantes dizem aproveitar a vida Mais ou menos, uma quantidade semelhante a da Tabela $1(47,22 \%)$ que classifica como Boa a sua qualidade de vida. Todavia, não se pôde compreender o que é qualidade de vida e o que é aproveitá-la para cada um deles, isto é, não se desvelou o que cada uma dessas premissas significa sob sua opinião.

Com relação à Tabela 1, fica evidente certo equilíbrio sobre as considerações sobre qualidade de vida. Isso pode ser entendido tendo em vista as diferenças de entendimento do conceito e dos interesses e variáveis mais relevantes, quanto ao assunto, para cada participante e sua respectiva análise subjetiva.

Trentini, Xavier e Fleck (2006) definem qualidade de vida como a percepção de um indivíduo quanto a sua posição na vida no que tange a seus valores, concepções e conceitos socioculturais relativos à suas metas, objetivos, preocupações e padrões. Dentro dessa definição, Freitas (2020) diz que o bem-estar provém do sentido existencial atribuído à vida, o qual eleva em 33\% os sentimentos e atitudes relativas à: aceitação de si mesmo, relações interpessoais sadias, crescimento pessoal, domínio do ambiente e propósito individual.

Tabela 2 - Aproveitamento da vida

\begin{tabular}{lcc}
\hline Aproveitamento & F & \% \\
\hline Nada & 2 & 5,55 \\
Muito pouco & 8 & 22,22 \\
Mais ou menos & 16 & 44,44 \\
Bastante & 8 & 22,22 \\
Extremamente & 2 & 5,55 \\
\hline Total & $\mathbf{3 6}$ & $\mathbf{1 0 0}$ \\
\hline
\end{tabular}

Quanto à questão levantada sobre a vida dos idosos, nota-se, na Tabela 2, que a maior parcela $(44,44 \%)$ dos entrevistados disse que a aproveita Mais ou menos. Além disso, 22,22\% dos participantes responderam que aproveitam Muito pouco e outros 22,22\% Bastante. A porcentagem 5,55\% se repete ainda com relação à escolha das opções Nada e Extremamente, respectivamente. 
Para verificar se há diferença de significância, aplicou-se o teste do Qui-quadrado. Descobriu-se que $\chi \mathrm{o}^{2}=3,99$ e $\chi^{2}=9,48$, não existindo diferença estatisticamente significante. Sabe-se ainda que n.g.l.=4 e $\alpha=0,05$.

Quanto aos resultados, é nítido o equilíbrio existente entre as opiniões, sendo interessante discutir algumas possibilidades de entendimento. Aproveitamento da vida é um tema genérico e perpassa, individual e subjetivamente, interesses, opiniões e projetos diversos.

Martins et al. (2007) dizem que os idosos precisam usar estratégias que os possibilitem ter uma velhice satisfatória, o que implica em mudar seus hábitos, engajar-se em atividades produtivas, realizar seus projetos de vida, ingressar numa universidade de terceira idade, desenvolver serviços voluntários, dentre tantas outras iniciativas possíveis. Um envelhecimento bem-sucedido depende de como o idoso enfrentará os desafios da vida, lutará pelos seus direitos e colocará em prática projetos viáveis dentro das condições pessoais e do ambiente em que vive.

Freitas (2020) completa ao dizer que possuem um sentido definido para suas vidas têm mais habilidades sociais e estão mais abertos para discernir e buscar o apoio adequado quando precisam, pois o bem-estar resulta desse sentido existencial, o qual influi no desenvolvimento psicológico e na forma como os indivíduos enfrentam seus desafios.

Neri (2001) finaliza ao dizer que a satisfação com a vida é uma das medidas do bem-estar psicológico que reflete a avaliação pessoal do indivíduo sobre determinados domínios. Um aspecto essencial do bem-estar psicológico é a assimilação de informações positivas sobre o self, um sistema composto por estruturas de conhecimento sobre si mesmo e um conjunto de funções cognitivas que integram ativamente essas mesmas estruturas ao longo do tempo e de várias áreas do funcionamento pessoal.

Tabela 3 - Satisfação com o acesso aos serviços de saúde

\begin{tabular}{lcc}
\hline Satisfação & F & \% \\
\hline Muito insatisfeito & 1 & 2,77 \\
Insatisfeito & 4 & 11,11 \\
Nem satisfeito nem insatisfeito & 9 & 25 \\
Satisfeito & 15 & 41,66 \\
Muito satisfeito & 6 & 16,66 \\
\hline Total & $\mathbf{3 5}$ & $\mathbf{1 0 0}$ \\
\hline
\end{tabular}

É constatado na Tabela 3 que a maior parcela dos sujeitos entrevistados (41,66\%) está Satisfeita com seu acesso aos serviços de saúde, além de $25 \%$ que se dizem Nem satisfeitos nem insatisfeitos. Ainda há 16,66\% que se dizem Muito satisfeitos. Em oposição, 11,11\% dos idosos optaram pela resposta Insatisfeito e outros 2,77\% por Muito insatisfeito. 
Frisa-se que $\chi \mathrm{O}^{2}=4,20$ e $\chi^{2}=9,48$, já que o teste não paramétrico do Qui-quadrado foi aplicado com o intuito de averiguar se existe ou não diferença estatisticamente significante; verificou-se que não existe tal diferença. Salienta-se que n.g.l. $=4$ e $\alpha=0,05$.

Colalto (2002) diz que apenas 40\% dos idosos se encontram satisfeitos com os serviços de saúde que possuem. Esses dados são congruentes com os da Tabela 3, que diz que 41,66\% dos voluntários se consideram Satisfeitos com seu acesso aos mesmos serviços.

Trentini, Xavier e Fleck (2006) dizem que o estado de velhice bem-sucedida se dá quando há a preservação do estado físico de saúde como dos jovens, tomando um estado bom ou ótimo com respeito ao bem-estar social e pessoal como exemplo. Essas características são atribuídas à maioria dos participantes mediante seu ponto de vista.

Porém, segundo Beres (1994), os idosos estão unanimemente insatisfeitos com o atendimento médico, uma vez que os profissionais não lhes deixam falar, não fazem perguntas e, às vezes, nem sequer levantam o olhar a eles.

Tabela 4 - Satisfação com o meio de transporte

\begin{tabular}{lcc}
\hline Satisfação & F & \% \\
\hline Muito insatisfeito & 3 & 8,33 \\
Nem satisfeito nem insatisfeito & 8 & 22,22 \\
Satisfeito & 13 & 36,11 \\
Muito satisfeito & 7 & 19,44 \\
\hline Total & $\mathbf{3 6}$ & $\mathbf{1 0 0}$ \\
\hline
\end{tabular}

Os resultados encontrados em nossa pesquisa mostram através da Tabela 4 que 36,11\% dos participantes encontram-se Satisfeitos e 22,22\% Nem satisfeitos nem insatisfeitos com o meio de transporte. Em contrapartida, além dos 19,44\% dos entrevistados que optaram pela afirmação Muito satisfeito, 8,33\% se mostraram Muito insatisfeitos. Nenhum idoso optou pela variável Insatisfeito, o que fez com que esta variável fosse eliminada da tabela.

Para verificar se há diferença estatisticamente significante, aplicou-se o teste do Qui-quadrado. Descobriu-se que $\chi \mathrm{o}^{2}=2,20$ e $\chi_{c}^{2}=7,81$, não existindo, assim, diferença estatisticamente significante. Além disso, considera-se n.g.l. $=3$ e $\alpha=0,05$.

Não foi possível verificar de forma pormenorizada qual o tipo de meio de transporte que os participantes utilizam e nem a sua frequência ou importância (para quais finalidades ou destinos eles os usam). 
Tabela 5 - Frequência de sentimentos negativos

\begin{tabular}{lcc}
\hline Assiduidade & F & \% \\
\hline Nunca & 7 & 19,44 \\
Algumas vezes & 23 & 58,33 \\
Frequentemente & 3 & 8,33 \\
Sempre & 3 & 8,33 \\
\hline Total & $\mathbf{3 6}$ & $\mathbf{1 0 0}$ \\
\hline
\end{tabular}

A Tabela 5 evidencia que, entre os resultados encontrados na pesquisa, pouco mais da metade (58,33\%) dos voluntários diz ter Algumas vezes sentimentos negativos, outros 19,44\% diz Nunca ter tais sentimentos e há dois grupos distintos de 8,33\% que optam pelas alternativas Frequentemente e Sempre. Nenhum idoso avaliou-se quanto a ter Muito frequentemente sentimentos negativos, o que fez com que esta variável fosse eliminada da tabela.

Vale ressaltar que $\chi \mathrm{o}^{2}=8,53$ e $\chi^{2}=7,81$; já que o teste do Qui-quadrado foi aplicado para verificar se existe diferença estatisticamente significante; verificou-se que tal diferença existe. Frisa-se que n.g.l.=3 e $\alpha=0,05$.

Zimerman (2000) diz que $15 \%$ dos idosos necessitam de atendimento em saúde mental e $2 \%$ apresentam quadros de depressão que, muitas vezes, não são percebidos pelos familiares e cuidadores, sendo encarados como características naturais da velhice.

Veras (1995) diz que, devido à velhice e as dificuldades sexuais causadas por essa fase do desenvolvimento humano, as mulheres podem passar a se achar menos atraentes e incapazes de conquistar seu marido, tendo conflitos emocionais. Já o homem, passa pela mesma causa que as mulheres, pode se sentir impotente e ter uma visão negativa do próprio corpo.

Freitas (2020) diz que o sentido e a apreciação da vida influenciam o bem-estar e aumentam em $33 \%$ os sentimentos e atitudes relativas à: aceitação de si, relações interpessoais sadias, crescimento pessoal, domínio do ambiente e propósito, embora diminuam bem pouco as emoções negativas. Por sua vez, o estado de tranquilidade quanto ao rumo tomado pela vida diminui as emoções negativas e também influencia no bem-estar.

Tabela 6 - Não recebimento de força pessoal e apoio de Deus

\begin{tabular}{lcc}
\hline Fortificação & F & \% \\
\hline Concordo Totalmente & 4 & 11,11 \\
Concordo Parcialmente & 2 & 5,55 \\
Concordo mais que discordo & 1 & 2,77 \\
Discordo mais que concordo & 1 & 2,77 \\
Discordo Parcialmente & 4 & 11,11 \\
Discordo Totalmente & 24 & 66,66 \\
\hline Total & $\mathbf{3 6}$ & $\mathbf{1 0 0}$ \\
\hline
\end{tabular}


Nota-se, na Tabela 6, que 66,66\% dos idosos entrevistados Discordam Totalmente da premissa de não receberem forças e apoio divino. Há dois grupos de $11,11 \%$ que Discordam Parcialmente e que Concordam Totalmente de tal suposição. Há ainda 5,55\% que Concordam Parcialmente e outros dois grupos de 2,77\% que Concordam mais que discordam e Discordam mais que concordam.

Com a intenção de descobrir se existe diferença estatisticamente significante, aplicou-se o teste do Qui-quadrado e obteve-se $\chi \mathrm{O}^{2}=0$ e $\chi_{c}^{2}=11,07$, não havendo diferença estatisticamente significante. É valido salientar também que n.g.l. $=5$ e $\alpha=0,05$.

Se o recebimento de apoio espiritual e força divina forem considerados como algo ligado à prática de orações, segundo Orlando et al. (2008), a maioria deles costuma pedir saúde, paz, amor, solução de problemas familiares, agradecer por graças alcançados, entre outras coisas. Vale frisar que o tipo de vida não altera suas petições.

De acordo com Diniz (2003), a religião é uma experiência que toca e modifica o ser humano. Ela não deve ser concreta - possuir credo e normas -, mas ser transformadora, pois se trata de uma fé que significa o encontro com Deus. Esse espaço sagrado é individual.

Segundo Araújo (1999), a religião tem total influência nessa fase da vida (já que $100 \%$ de seus pesquisados disseram apreciar alguma prática). Isso se dá por a religião facilitar a criação de um vínculo entre suas limitações e potencialidades; quando isso não ocorre, o idoso se sente ajudado a sobrepujar com maior facilidade essa etapa da vida.

Tabela 7 - Não apreciação da vida

\begin{tabular}{lcc}
\hline Desapreço & F & \% \\
\hline Concordo Totalmente & 1 & 2,77 \\
Concordo Parcialmente & 2 & 5,55 \\
Concordo mais que discordo & 1 & 2,77 \\
Discordo Parcialmente & 4 & 11,11 \\
Discordo Totalmente & 28 & 77,77 \\
\hline Total & $\mathbf{3 6}$ & $\mathbf{1 0 0}$ \\
\hline
\end{tabular}

Pode-se observar na Tabela 7 que 77,77\% dos sujeitos Discordam Totalmente da suposição de que não apreciam a vida. Apenas 11,11\% Discordam Parcialmente e 5,55\% Concordam Parcialmente. Há dois grupos de 2,77\% que Concordam Totalmente e Concordam mais que discordam respectivamente. A variável Discordo mais que concordo foi eliminada por não ter sido mencionada por nenhum participante.

Para verificar se existe diferença estatisticamente significante o teste do Qui-quadrado foi aplicado. Descobriu-se que $\chi \mathrm{o}^{2}=0$ e $\chi^{2}=9,48$, não existindo diferença estatisticamente significante; considera-se também que n.g.1.=4 e $\alpha=0,05$.

Freitas (2020) diz que o sentido e a apreciação da vida influenciam o bem-estar e acrescem em 33\% os sentimentos e atitudes relativas à: aceitação de si, relações interpessoais sadias, crescimento pessoal, 
domínio do ambiente e propósito. Os indivíduos que encontram sentido em suas vidas têm mais facilidade com habilidades sociais e em buscar o suporte necessário quando precisam, já que este mesmo sentido é responsável por afetar sensível e positivamente no desenvolvimento psicológico humano e no modo como os sujeitos enfrentam seus desafios.

Por sua vez, Panzini et al. (2007) dizem que o propósito da vida e o bem-estar estão associados à presença em serviços religiosos. As crenças influenciam de forma significativa na visão que o sujeito tem do mundo, de modo a promover felicidade.

\section{CONCLUSÃO}

Conclui-se que os idosos trabalhadores possuem uma qualidade de vida considerada como Boa em todos os aspectos biopsicossociais observados, segundo sua própria opinião. Porém, não foi possível verificar se o trabalho tem alguma influência nos campos de suas vidas, assim como houve questões que não puderam ser analisadas de forma mais aprofundada pelo material utilizado, como as finalidades e frequência de uso do meio de transporte pelos participantes.

Sugere-se que uma pesquisa seja realizada com idosos que não trabalham para se comparar os resultados com os desse artigo, bem como que as questões não respondidas sejam devidamente investigadas em um novo trabalho que seja mais específico para as temáticas.

Percebe-se ainda que o índice de religiosidade é altíssimo em relação às demais facetas investigadas e que esse aspecto deve ter um papel importante na vida dos idosos por fazer com que eles se sintam bem e seguros. 


\section{REFERÊNCIAS}

Araújo, C. D. S. F. (1999) Aspectos religiosos do idoso. In: Petroiano, A., Pimenta L. G. Org: Clínica e cirurgia geriátrica. p. 8-9. Rio de Janeiro: Guanabara.

Baptista, A. L. (2003) Espiritualidade na prática clínica. In: Arte Terapia Coleção Imagens da Transformação. Revista POMAR. 08, v.10, p. 18-23. Rio de Janeiro.

Bee, H. (1997) O ciclo vital. (Garcez, R., trans.). Porto Alegre: Artes Médicas.

Beres, V, G. (1994) “Agente que tem o amarelão tem que se conformar”: Atenção à saúde na perspectiva do idoso. Dissertação de mestrado, Curso de Psicologia, Núcleo de Psicologia Social - Universidade Católica de São Paulo, 76, São Paulo, Brasil.

Colalto, R. M. C. (2002) Qualidade de vida na terceira idade. Recuperado em 13 de abril, 2021, de Universidade Camilo Castelo Branco. Monografia como pré-requisito de Trabalho de Conclusão de Curso de graduação em Psicologia - p. 49. São Paulo, Brasil.

Diniz, L. (2003) Espiritualidade e Arte Terapia. In: Arte Terapia Coleção Imagens da Transformação. Revista POMAR. 10, v.10, 109-124. Rio de Janeiro.

Freitas, L. H. W. de (2020). A influência do sentido da vida sobre o bem estar. Archives of Health, 1(6), 432-447. https://doi.org/10.46919/archv1n6-006.

Martins, J. J.; Albuquerque, G. L.; Nascimento, E. R. P.; Barra, D. C. C.; Souza, W. G. A. e Pacheco, W. N. S. (2007) Necessidades de educação em saúde dos cuidadores de pessoas idosas no domicílio. Recuperado em 05 de maio, 2021, de http://www.scielo.br/scielo.

Neri, A. L. (2001) Maturidade e velhice (1ed.) Campinas: Papirus.

Orlando, C.; Dias, J. C.; Brasil, R. T.; Araújo, T. C.; Buriti, M. A. (2008) Religiosidade na dimensão biopsicossocial do sujeito idoso. Recuperado em 28 de abril, 2021, de Universidade São Judas Tadeu. Artigo apresentado no XIV Simpósio Multidisciplinar da USJT realizado em 2008. São Paulo.

Panzini, R. G.; Rocha, N. C.; Bandeira D. R.; Fleck, M. P. A. (2007) Qualidade de Vida e Espiritualidade. Org: Revista Psicologia clinica. 34, p.105-115.

Rabelo, D. F.; Souza, A. P.; Gomes, A. C.; Fujimoto, B. H. S.; Mota, J. J. O.; Oliveira, L. S.; et al. (2008) Atitudes de universitários da área da saúde em relação ao idoso e à velhice. Recuperado em 27 de novembro, 2008, de Centro Universitário de Patos de Minas - UNIPAM. Artigo apresentado na XXXVIII Reunião Anual de Psicologia da Sociedade Brasileira de Psicologia realizada em 2008. Uberlândia.

Trentini, C. M.; Xavier, F. M. F.; Fleck, M. P. A. (2006) Qualidade de vida em idosos. In: Parente, M. A. M. P. Cognição e envelhecimento. (1ed). p. 19-20. Porto Alegre: Artmed.

Veloz, M. C. T.; Nascimento-Schulze, C. M.; Camargo, B. V. (1999) Reflexões sociais do envelhecimento. Recuperado em 21 de abril, 2021, de http://www.scielo.br/scielo.

Zimerman, G. I. (2000) Velhice: Aspectos Biopsicossociais. Porto Alegre: Artmed. 